\title{
ADAPTIVE VISUAL SERVOING FOR CONSTRAINED ROBOTS UNDER JACOBIAN, JOINT DYNAMIC AND CONTACT VISCOUS FRICTION UNCERTAINTIES
}

\author{
E.C. Dean-León ${ }^{*, 1}$ V. Parra-Vega ${ }^{* *, 2}$ \\ L.G. García-Valdovinos ${ }^{*, 1}$ A.Espinosa-Romero ${ }^{* * * 3}$ \\ * Mechatronics Division, CINVESTAV - IPN \\ AP 14-740, México, D.F., 07300 México \\ ** Center for Advanced Technology - CIATEQ, México \\ *** Faculty of Mathematics, Univ. of Yucatán, México
}

\begin{abstract}
Visual servoing of constrained robots has not yet met a formal treatment nor its friction compensation. This kind of robots moves slowly along the constrained surface due to technological limitations of the camera system, therefore important problems of friction at the joint and contact point arise. The problem turns very complicated when parametric uncertainty on robot, camera and friction is considered. In this paper, a new visual servoing scheme that satisfy this problem is presented. It induces sliding modes without chattering to guarantee locally exponential convergence of tracking errors. Simulations results are presented and discussed. Copyright (c) 2005 IFAC
\end{abstract}

Keywords: Visual Servoing, Adaptive Control, Force Control, Uncertain Jacobian, Friction.

\section{INTRODUCTION}

It is well known that multisensor-based robot control approaches may offer a solution to very important and relevant, but complex, problems in robotics. In order to achieve this sensor fusionbased controller, a careful analysis of the dynamics, sensors behavior, and tasks are required. One of such tasks, is the image-based force-position control of robots. Furthermore, since parameters are in practice uncertain, the control must be robust to all parameters of the system. This sort of control laws are able to interact under changing environment and deal with uncertainties of its

\footnotetext{
1 Email: (edean,Igarcia)@cinvestav.mx

2 Partially supported by CONACYT project 39727-Y. On a sabbatical leave from Mechatronics Division, CINVESTAV. Email: vicente.parra@ciateq.mx

3 Email: eromero@tunku.uady. $m x$
}

dynamic models without the explicit intervention of humans. Concretely, the desired task is that the robot end effector tracks a visual trajectory along the surface of an object, and at the same time, control the applied force, see Figure 1.

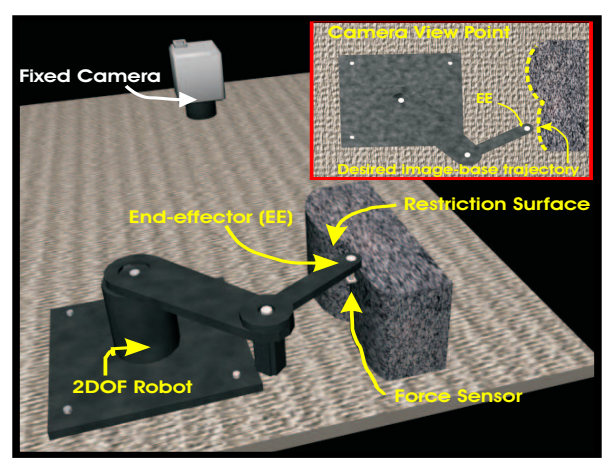

Figure 1. Robot-Force-Vision System. 
On the other hand, joint friction and contact friction are quite important to compensate in any practical application. Therefore, we consider for the joint friction the LuGre model, which reproduces a typical motion regime of image-based robotic tasks, and viscous friction at the contact point.

\subsection{Background}

Good sensing abilities of relevant variables are essential to gain higher flexibility and autonomy of robots in an unknown working environment. Often, either vision or force sensor are used. Hybrid vision/force control approaches have been reported e.g. (J. Baeten, 2000), (Xiao, 2000) and none of them shows robustness to uncertainties, neither robot parameters nor camera parameters.

With respect to force control, Arimoto (Arimoto, 1996) solved by first time the simultaneous control of position and force using the full nonlinear dynamics subject to parametric uncertainties without coordinate partitioning, based on the orthogonalization principle. Afterwards, several schemes have been proposed based on the orthogonalization principle, however these schemes have not been extended or combined with visual servoing beyond free motion robots.

In this paper, an adaptive controller driven by image and contact force errors to solve the problem posed above is proposed. The system guarantees exponential tracking of position and force trajectories subject to parametric uncertainties. This scheme delivers a smooth controller and presents formal stability proofs. Simulations allows to visualize the expected closed loop performance predicted by the theory.

\section{NONLINEAR ROBOT DYNAMICS}

The constrained robot dynamics arises when its end effector is in contact with infinitely rigid surface. Considering the generalized position $q \in$ $R^{n}$ and velocity coordinates $\dot{q} \in R^{n}$, this system is (Arimoto, 1996)

$$
\begin{aligned}
H(q) \ddot{q}+C(q, \dot{q}) \dot{q}+g(q)= & \tau+J_{\varphi+}^{T}(q) \lambda-F(\dot{q}, \psi) \\
& -J^{T}(q) B_{t} J(q) \dot{q} \\
\varphi(q) & =0
\end{aligned}
$$

where $B_{t} \in R_{+}^{n \times n}$, is the viscous friction matrix and possibly not a diagonal matrix, $J^{T}(q) B_{t} J(q) \dot{q}$ represents the tangencial viscous friction in the contact point, $H(q) \in R^{n \times n}$ stands for the inertia matrix; $C(q, \dot{q}) \dot{q} \in R^{n}$ stands for the vector of centripetal and Coriolis torques; $g(q) \in R^{n}$ is the vector of gravitational torques, $F(\dot{q}, \psi)$ is the joint dynamic friction ${ }^{4}, J_{\varphi}(q)$ is constrained jacobian of the rigid and frictionless constraint surface $\varphi(q)=0$, and $\lambda$ is the constrained Lagrangian or contact force.

Adding and subtracting to (1) the linear parametrization $H(q) \ddot{q}_{r}+C(q, \dot{q}) \dot{q}_{r}+g(q)+J^{T}(q) B_{t} J(q) \dot{q}=$ $Y_{r} \theta_{b}$, where the known regressor $Y_{b}=Y_{b}\left(q, \dot{q}, \dot{q}_{r}, \ddot{q}_{r}\right) \in$ $\Re^{n \times p}$ and the unknown constant vector $\theta_{b} \in \Re^{p}$, produces the open loop error equation

$$
\begin{gathered}
H(q) \dot{S}_{q}=-C(q, \dot{q}) S_{q}-J^{T}(q) B_{t} J(q) S_{q}+ \\
\tau+J_{\varphi}^{T}(q) \lambda-Y_{r} \theta_{b}
\end{gathered}
$$

with joint error surface $S_{q}$ is defined as

$$
S_{q}=\dot{q}-\dot{q}_{r}
$$

where $\dot{q}_{r}$ stands for the nominal reference.

\section{CAMERA MODEL}

The static pin hole with thin lens without aberration camera model is used (S. Hutchinson, 1996). To introduce it, consider the forward kinematics $x_{b}=f(q)$, for $x_{b} \in \Re^{n}$ represents the position of robot end effector in cartesian space. Differential kinematics relates velocities in cartesian space to joint space velocities as follows $\dot{x}_{b}=J(q) \dot{q}$. According to (S. Hutchinson, 1996), visual position $x_{s} \in \Re^{2}$ of robot end effector in image space is given by $x_{s}=\alpha R(\theta) x_{b}+\beta$ where $\alpha$ is the scale factor $^{5}, R(\theta) \in S O(3), \beta \in \Re^{2}$ that depends on intrinsic and extrinsic camera parameters. The differential kinematics of camera model is then $\dot{x}_{s}=\alpha R(\theta) \dot{x}_{b}$. Using these relationships, we have an equation that relates $\dot{x}_{s} \in \Re^{2}$ and $\dot{q} \in \Re^{2}$ as follows:

$$
\dot{x}_{s}=\alpha R(\theta) J(q) \dot{q} \Longrightarrow \dot{q}=J_{R i n v} \dot{x}_{s}
$$

whit $J_{R i n v}=J(q)^{-1} R(\theta)^{-1} \alpha^{-1}$. This relation is useful to design the nominal reference.

\section{VISUAL-FORCE EXTENDED ERROR}

Since $\varphi(q)=0 \forall t$, then its time derivative yields $\frac{d}{d t} \varphi(q)=\frac{\partial \varphi(q)}{\partial q} \frac{d q}{d t} \equiv J_{\varphi}(q) \dot{q}=0$, this means that $J_{\varphi}(q)$ is orthogonal to $\dot{q}$. That is, $\dot{q}$ belongs to the orthogonal projection matrix $Q$ of $J_{\varphi}(q)$ (Arimoto, 1996)

$$
Q=I-\frac{J_{\varphi}}{\left\|J_{\varphi}(x)\right\|^{2}} J_{\varphi}^{T}
$$

As we can see, $Q$ spans the tangent plane at the contact point, therefore, $J_{\varphi}$ and $Q$ are orthogonal complements. In other words, $Q \dot{q}=\dot{q} \rightarrow Q Q \dot{q}=$

\footnotetext{
4 For a clear exposition, firstly, $F(\dot{q}, \psi)$ will be considered zero, however in Section 7 it will be treated.

5 Without loss of generality, $\alpha$ can be considered as a scalar matrix $2 \times 2$.
} 
$Q \dot{q} \equiv \dot{q}$, naturally, $Q J_{\varphi}^{T}=0$. These properties are fundamental to establish the visual orthogonalization principle. Now, let $\dot{q}_{r}$, in (4), be constructed in terms of orthogonal nominal references of velocity $\dot{q}_{s}$ and force $\dot{q}_{f}$ as follows

$$
\dot{q}_{r}=\dot{q}_{s}+\dot{q}_{f} \equiv Q J_{R i n v} \dot{x}_{r}+\beta J_{\varphi}^{T} \dot{q}_{r f}
$$

Furthermore, let the nominal visual reference $\dot{x}_{r}$ be

$$
\dot{x}_{r}=\dot{x}_{s d}-\alpha \Delta x_{s}+S_{s d}-\gamma_{s} \int_{t_{0}}^{t} \operatorname{sign}\left(S_{s \delta}\right)
$$

where $\dot{x}_{s d}$ stands for desired visual velocity trajectory and $\Delta x_{s}=x_{s}-x_{s d}$ is the visual position error, and $S_{s}=\Delta \dot{x}_{s}+\alpha \Delta x_{s}, S_{s d}=S_{s}\left(t_{0}\right) e^{-\kappa_{s} t}$, $S_{s \delta}=S_{s}-S_{s d}$, where $\Delta \dot{x}_{s}=\dot{x}_{s}-\dot{x}_{s d}, \kappa_{s}>0$, and $\gamma_{s} \in R^{n \times n}$. Now let us design the nominal force reference as follows

$$
\dot{q}_{r f}=\Delta F-S_{d F}+\gamma_{F} \int_{t_{0}}^{t} \operatorname{sign}\left(S_{F \delta}\right)
$$

where $S_{F}=\Delta F, S_{F d}=S_{F}\left(t_{0}\right) e^{-\kappa_{F} t}, S_{F \delta}=S_{F}-$ $S_{F d}$, with $\Delta F=\int_{t_{0}}^{t} \Delta \lambda(\zeta) d \zeta, \Delta \lambda=\lambda-\lambda_{d}$, and $\lambda_{d}$ is the desired contact force, $\kappa_{F}>0$, $\gamma_{F} \in R_{+}^{n \times n}$.

Using equations (7) into (4), we obtain the following orthogonalized joint error surface ${ }^{6}$

$$
\begin{aligned}
S_{q} & =\dot{q}-\dot{q}_{r} \equiv Q \dot{q}-\dot{q}_{r} \\
& =Q J_{R i n v} \dot{x}_{s}-Q J_{R i n v} \dot{x}_{r}-\beta J_{\varphi}^{T} \dot{q}_{r f} \\
& =Q J_{R i n v} S_{v s}-\beta J_{\varphi}^{T} S_{v F}
\end{aligned}
$$

with $S_{v F}=S_{F \delta}+\gamma_{F} \int_{t_{0}}^{t} \operatorname{sign}\left(S_{F \delta}\right)$ and $S_{v s}=$ $S_{s \delta}+\gamma_{s} \int_{t_{0}}^{t} \operatorname{sign}\left(S_{s \delta}\right)$.

Remark 1. The above definition assumes exact knowledge of $J_{\text {Rinv }}$, however, in practice it is a very restricted assumption. Therefore, we need to design uncertain manifold $S_{q}$ taking into consideration uncertainty of $J_{q}, R(\theta), \alpha$.

To this end, consider

$$
\widehat{\dot{q}}_{r}=Q \widehat{J_{R i n v}} \dot{x}_{r}+\beta J_{\varphi}^{T} \dot{q}_{r f}
$$

with $\widehat{J_{\text {Rinv }}}$ an estimated of $J_{\text {Rinv }}$, such as rank $J^{-1}(q)$ and $R_{\alpha}^{-1}(\theta)$ are full rank $\forall q \in \Omega$, where the robot workspace free of singularities is defined by $\Omega=\{q \mid \operatorname{rank}(J(q))=n\}$, and $\forall \theta \in \Re$. Thus, substituting (11) into (4) we have the uncalibrated joint error surface

$$
\hat{S}_{q}=\dot{q}-\widehat{\dot{q}}_{r}=Q J_{R i n v} \dot{x}_{s}-Q \widehat{J_{R i n v}} \dot{x}_{r}-\beta J_{\varphi}^{T} \dot{q}_{r f}
$$

where $\hat{S}_{q}$ is available because $\dot{q}$ and $\widehat{\dot{q}}_{r}$ are available.

\footnotetext{
6 Notice that $S_{q}$ is composed of two orthogonal complements, $Q J_{R i n v} S_{v s}$ depending on image coordinate error, and $\beta J_{\varphi}^{T} S_{v F}$ depending of integral of contact force errors.
}

\section{OPEN LOOP ERROR EQUATION}

Using (11), the uncertain parametrization is $H(q) \widehat{\ddot{q}}_{r}+C(q, \dot{q}) \hat{\dot{q}}_{r}+g(q)+J^{T}(q) B_{t} J(q) \hat{\dot{q}}_{r}=Y_{r} \hat{\theta}_{b}$ where $\ddot{q}_{r}=f\left(\ddot{x}_{r}, \ddot{q}_{f r}\right)$, and $\ddot{x}_{r}=\ddot{x}_{s d}-\alpha \Delta \dot{x}_{s}+$ $\dot{S}_{s d}-\gamma_{s} \operatorname{sign}\left(S_{s \delta}\right), \ddot{q}_{r f}=\Delta \dot{F}-\dot{S}_{d F}+\gamma_{F} \operatorname{sign}\left(S_{F \delta}\right)$, which produces a discontinuous $\ddot{q}_{r}$.

To avoid introducing high frequency discontinuous signals, add and subtract $z_{s}=\tanh \left(\nu_{s} S_{s \delta}\right)$ and $\tanh \left(\nu_{f} S_{F \delta}\right)$ to $\ddot{q}_{r}$ to separate continuous and discontinuous signals as follows

$$
\ddot{q}_{r}=\ddot{q}_{\text {cont }}+Q \gamma_{s} z_{s}-\beta J_{\varphi}^{T} \gamma_{f} z_{f}
$$

with $z_{s}=\tanh \left(\lambda_{s} S_{s \delta}\right)-\operatorname{sign}\left(S_{s \delta}\right)$ and $z_{f}=$ $\tanh \left(\lambda_{f} S_{F \delta}\right)-\operatorname{sign}\left(S_{F \delta}\right)$. This results that $Y_{\text {cont }}=$ $Y_{r}\left(q, \dot{q}, \dot{q}_{r}, \ddot{q}_{\text {cont }}\right)$ is continuous since $\left(\dot{q}_{r}, \ddot{q}_{\text {cont }}\right) \in$ $\mathcal{C}^{1}$, where

$$
\begin{aligned}
\widehat{\ddot{q}}_{\text {cont }}= & Q{\widehat{J_{R i n v}}}_{\ddot{x}_{c o n t}}+\beta J_{\varphi}^{T} \ddot{q}_{c o n t}+ \\
& \dot{Q} \widehat{J_{\text {Rinv }}} \dot{x}_{s}+\beta \dot{J}_{\varphi}^{T} \dot{q}_{r f}
\end{aligned}
$$

with $\ddot{x}_{c o n t}=\ddot{x}_{s d}-\alpha \Delta \dot{x}_{s}+\dot{S}_{s d}+\gamma_{s} \tanh \left(\nu_{s} S_{s \delta}\right)$, $\ddot{q}_{\text {cont }}=\Delta \dot{F}-\dot{S}_{d F}+\gamma_{F} \tanh \left(\nu_{f} S_{F \delta}\right)$. It will become later that large $\nu_{s}, \nu_{f}$ are not required in the control law design to induce sliding modes. Therefor the uncalibrated linear parametrization is

$$
\begin{aligned}
& H(q) \widehat{\ddot{q}_{r}}+C(q, \dot{q}) \widehat{\dot{q}_{r}}+g(q)+J^{T}(q) B_{t} J(q) \widehat{\dot{q}_{r}}= \\
& Y_{\text {cont }} \hat{\theta}_{b}+H\left(Q \gamma_{s} z_{s}-\beta J_{\varphi}^{T} \gamma_{f} z_{f}\right)
\end{aligned}
$$

where the last term can be seen as a disturbance.

Adding and subtracting (15) to (1) we obtain finally the open loop error equation.

$$
\begin{gathered}
H(q) \widehat{\dot{S}_{q}}=-C(q, \dot{q}) \widehat{S_{q}}+\tau+J_{\varphi}^{T}(q) \lambda- \\
Y_{\text {cont }} \theta_{b}-J^{T}(q) B_{t} J(q) \widehat{S_{r}}+ \\
H\left(Q \gamma_{s} z_{s}-\beta J_{\varphi}^{T} \gamma_{f} z_{f}\right)
\end{gathered}
$$

Now we are ready to present the main result.

\section{CONTROL DESIGN}

Theorem 1: Assume that initial conditions and desired trajectories belong to $\Omega$. Consider the constrained robot dynamics (1), subject to parametric uncertainties camera, robot and tangential contact friction, in closed loop with the following visual adaptive force-position control law

$$
\begin{gathered}
\tau=-K_{d} \hat{S}_{q}+\bar{Y}_{\text {cont }} \hat{\theta}_{b}+J_{\varphi}^{T}(q)\left[-\lambda_{d}+\eta \Delta F\right]+ \\
\gamma_{F} J_{\varphi}^{T}(q)\left[\tanh \left(\mu S_{q F}\right)+\eta \int_{t_{0}}^{t} \operatorname{sgn}\left(s_{F \delta}\right)\right] \\
\dot{\hat{\theta}}_{b}=-\Gamma Y_{\text {cont }}^{T} \hat{S}_{q}
\end{gathered}
$$

where $\Gamma \in R_{+}^{p \times p}, K_{d} \in R_{+}^{n \times n}, \eta>0$, and $\lambda_{d}$ the desired contact force. If $K_{d}$ is large enough and an 
error of initial conditions are small enough, with $\gamma_{s} \geq\left\|\frac{d}{d t}\left\{R_{\alpha}(\theta) J(q)\left[\hat{S}_{q}+\left(\widehat{J_{\text {Rinv }}}-J_{\text {Rinv }}\right) \dot{x}_{r}\right]\right\}\right\|$ and $\gamma_{F} \geq\left\|\frac{d}{d t}\left[\left(J_{\varphi} J_{\varphi}^{T}(q)\right)^{-1} J_{\varphi} \hat{S}_{q}\right]\right\|$ then exponential convergence of visual and force tracking errors is guaranteed.

Proof: We prove that the closed loop dynamics (CLD) between (17)-(18) and (1), shows boundedness of all system trajectories, with exponential convergence of tracking errors. The proof is organized in three parts.

Part I. Boundedness of Closed Loop Trajectories. Consider the time derivative of the following Lyapunov candidate function

$$
V=\frac{1}{2}\left[\hat{S}_{q}^{T} H(q) \hat{S}_{q}+\beta S_{v F}^{T} S_{v F}+\Delta \theta_{b}^{T} \Gamma \Delta \theta_{b}\right],
$$

with $\Delta \theta_{b}=\theta_{b}-\hat{\theta}$, along the solutions of closed loop dynamics (CLD) as

$$
\dot{V} \leq-K_{d}\left\|\hat{S}_{q}\right\|_{2}^{2}-\eta \beta\left\|S_{v F}\right\|+\left\|\hat{S}_{q}\right\| \psi
$$

where $\psi$ is a functional depending on the state and error manifolds. Now if $K_{d}, \eta$ and $\beta$ are large enough and the initial errors are small enough, we conclude the seminegative definiteness of $\dot{V}$ outside of hyperball $\varepsilon_{0}=\left\{\widehat{S_{q}} \mid \dot{V} \geq 0\right\}$ centered at the origin, such as the following properties of the state of closed loop system arise

$$
\widehat{S_{q}}, S_{v F} \in \mathcal{L}_{\infty} \rightarrow\left\|S_{v s}\right\|,\left\|S_{v F}\right\| \in \mathcal{L}_{\infty}
$$

Then, $\left(S_{s \delta}, \int \operatorname{sign}\left(S_{s \delta}\right)\right) \in \mathcal{L}_{\infty}$ and since desired trajectories are $C^{2}$ and feedback gains are bounded, we have that $\left(\widehat{\dot{q}}_{r}, \widehat{\ddot{q}}_{r}\right) \in \mathcal{L}_{\infty}$. The right hand side of (16) shows that $\varepsilon_{1}>0$ exists such that

$$
\left\|\widehat{\dot{S}}_{q}\right\| \leq \varepsilon_{1}
$$

This result shows only local stability of $\widehat{S_{q}}$ and $\widehat{\dot{S}_{q}}$, now we prove that the sliding modes arises. Adding and subtracting $Q J_{R i n v} \dot{x}_{r}$ to (12) we obtain

$$
\hat{S}_{q}=Q\left\{J_{\text {Rinv }} S_{v s}-\Delta J_{R i n v} \dot{x}_{r}\right\}-\beta J_{\varphi}^{T}\left\{S_{v F}\right\}
$$

where $\Delta J_{\text {Rinv }}=\widehat{J_{\text {Rinv }}}-J_{\text {Rinv }}$. Since $\hat{S}_{q} \in \mathcal{L}_{2}$, and $J_{\text {Rinv }}$ and $Q$ are bounded, then $Q J_{\text {Rinv }} S_{v s}$ is bounded and, due to $\varphi(q)$ is smooth and lies in the reachable robot space and $S_{v F} \rightarrow 0$, then $\beta J_{\varphi}^{T} S_{v F} \rightarrow 0$. Now, taking into account that $\widehat{\dot{S}}_{q}$ is bounded, then $\frac{d}{d t} J_{R i n v} Q S_{v s}$ and $\frac{d}{d t} \beta J_{\varphi}^{T} S_{v F}$ are bounded (this is possible because $\dot{J}_{\varphi}^{T}$ is bounded and so $\dot{Q}$ is). All this chains of conclusions proves that there exists constants $\varepsilon_{2}>0$ and $\varepsilon_{3}>0$ such that $\left|\dot{S}_{v s}\right|<\varepsilon_{2},\left|\dot{S}_{v F}\right|<\varepsilon_{3}$. Now, we have to prove that for a proper selection of feedback gains $\gamma_{s}$ and $\gamma_{F}$, we can conclude that trajectories of visual position and force converges to zero. This is possible if we can prove that sliding modes are established for the subspace of visual position $Q$ and the subspace of force $J_{\varphi}^{T}(q)$. Considering that operator $Q J_{\text {Rinv }}$ spans the vector $\hat{S}_{q}$ in its image $\operatorname{im}\left\{Q J_{\text {Rinv }}\left(S_{v s}\right)\right\} \equiv S_{v s}^{i m}$ and the operator $\beta J_{\varphi}^{T}$ spans the same vector in its image $i m\left\{\beta J_{\varphi}^{T}\left(S_{v F}\right)\right\} \equiv S_{v F}^{i m}$, this implies that

$$
\begin{aligned}
\hat{S}_{q} & =Q\left\{J_{R i n v} S_{v s}-\Delta J_{R i n v} \dot{x}_{r}\right\}-\beta J_{\varphi}^{T}\left\{S_{v F}\right\} \\
& =\left(S_{v s}^{i m}-i m\left\{\Delta J_{R i n v} \dot{x}_{r}\right\}\right)-S_{v F}^{i m}
\end{aligned}
$$

where $S_{v s}^{i m}-i m\left\{\Delta J_{R i n v} \dot{x}_{r}\right\}$ and $S_{v F}^{i m}$ belongs to a orthogonal complements, $<S_{v s}^{i m}-i m\left\{\Delta J_{R i n v} \dot{x}_{r}\right\}$, $S_{v F}^{i m}>=0$. That is, we are able to analyze the $S_{v s}^{i m}-i m\left\{\Delta J_{R i n v} \dot{x}_{r}\right\}$ dynamics, independently of $S_{v F}^{i m}$, since $S_{v F}^{i m}$ belongs to the kernel of $Q$. This is verified if we multiply (21) for $Q^{T}$,

$$
\begin{aligned}
Q^{T} \hat{S}_{q} & =Q^{T} Q\left\{J_{R i n v} S_{v s}-\Delta J_{R i n v} \dot{x}_{r}\right\}-\beta Q^{T} J_{\varphi}^{T} S_{v F} \\
& =S_{v s}^{\text {im }}-i m\left\{\Delta J_{\text {Rinv }} \dot{x}_{r}\right\}
\end{aligned}
$$

since $Q$ is idempotent $\left(Q^{T} Q=Q\right)$. It is important to notice that if $A x=A y$ for any square nonsingular matrix $A$ and any couple of vectors $x, y$, then $x \equiv y$. Thus, the equation (22) means that for the subspace $Q$, the equality $\hat{S}_{q}=$ $Q\left\{J_{R i n v} S_{v s}-\Delta J_{R i n v} \dot{x}_{r}\right\}$ is valid within span of $Q$. Now, if we multiply $\hat{S}_{q}$ for $J_{\varphi}$ to obtain

$$
\begin{aligned}
J_{\varphi} \hat{S}_{q} & =J_{\varphi} Q\left\{J_{R i n v} S_{v s}-\Delta J_{R i n v} \dot{x}_{r}\right\}-\beta J_{\varphi} J_{\varphi}^{T}\left\{S_{v F}\right\} \\
& =S_{v F}^{i m}
\end{aligned}
$$

\section{Part II: Second Order Sliding Modes.}

Part II.a: Sliding modes for the velocity subspace $Q$. According to $Q^{T} \hat{S}_{q}=Q\left\{J_{R i n v} S_{v s}-\right.$ $\left.\Delta J_{R i n v} \dot{x}_{r}\right\}$, then $\hat{S}_{q} \equiv J_{\text {Rinv }} S_{v s}-\Delta J_{\text {Rinv }} \dot{x}_{r}$ in the subspace image of $Q$, however notice that $Q$ is not full rank, then this equality is valid locally, not globally. In this local neighborhood, if we multiply the equality $\hat{S}_{q}=Q\left\{J_{\text {Rinv }} S_{v s}-\Delta J_{\text {Rinv }} \dot{x}_{r}\right\}$ by $R_{\alpha}(\theta) J(q)^{7}$, we have

$$
\begin{array}{r}
R_{\alpha}(\theta) J(q) \hat{S}_{q}=S_{s \delta}+\gamma_{s} \int \operatorname{sign}\left(S_{s \delta}\right)- \\
R_{\alpha}(\theta) J(q)\left\{\Delta J_{\text {Rinv }} \dot{x}_{r}\right\}
\end{array}
$$

Taking the time derivative of the above equation, and multiply it by $S_{s \delta}^{T}$ produces

$$
\begin{aligned}
S_{s \delta}^{T} \dot{S}_{s \delta} & =-\gamma_{s} S_{s \delta}^{T} \operatorname{sign}\left(S_{s \delta}\right)+ \\
& S_{s \delta}^{T} \frac{d}{d t}\left[R_{\alpha}(\theta) J(q)\left(\hat{S}_{q}+\Delta J_{R i n v} \dot{x}_{r}\right)\right] \\
& \leq-\mu_{s}\left|S_{s \delta}\right|
\end{aligned}
$$

where $\mu_{s}=\gamma_{s}-\varepsilon_{4}$, and $\varepsilon_{4}=\frac{d}{d t}\left[R_{\alpha}(\theta) J(q)\left(\hat{S}_{q}+\right.\right.$ $\left.\left.\Delta J_{\text {Rinv }} \dot{x}_{r}\right)\right]$. Thus, we obtain the sliding condition if $\gamma_{s}>\varepsilon_{4}$, such as, $\mu_{s}>0$ of (25) guarantee the sliding mode at $S_{s \delta}=0$ in a time $t_{s}=\frac{\left|S_{s \delta}\left(t_{0}\right)\right|}{\mu_{s}}$. However, notice that for any initial condition $S_{s \delta}\left(t_{0}\right)=0$, then $t_{s}=0$, which implies that the

\footnotetext{
7 Remember the equality: $J_{R i n v}=J^{-1}(q) R^{-1}(\theta) \alpha^{-1}$.
} 
sliding mode at $S_{s \delta}(t)=0$ is guaranteed for all time.

Part II.b: Sliding modes for the force subspace. Similarly, if we multiply (23) by $\left(J_{\varphi} J_{\varphi}^{T}(q)\right)^{-1}$, we obtain

$$
\begin{aligned}
\left(J_{\varphi} J_{\varphi}^{T}(q)\right)^{-1} J_{\varphi} \hat{S}_{q} & =-\beta J_{\varphi} J_{\varphi}^{T}\left\{S_{v F}\right\} \\
J_{\varphi}^{\#}(q) \hat{S}_{q} & =S_{F \delta}+\gamma_{F} \int \operatorname{sign}\left(S_{F \delta}\right)
\end{aligned}
$$

where $J_{\varphi}^{\#}(q)=\left(J_{\varphi} J_{\varphi}^{T}(q)\right)^{-1} J_{\varphi}$. Derivating the above equation and multiply for $S_{F \delta}^{T}$ lies

$$
\begin{aligned}
S_{F \delta}^{T} \dot{S}_{F \delta} & =-\gamma_{F}\left|S_{F \delta}\right|+S_{F \delta}^{T} \frac{d}{d t}\left(J_{\varphi}^{\#}(q) \hat{S}_{q}\right) \\
& \leq-\mu_{F}\left|S_{F \delta}\right|
\end{aligned}
$$

where $\mu_{F}=\gamma_{F}-\varepsilon_{5}$, and $\varepsilon_{5}=\frac{d}{d t}\left[\left(J_{\varphi} J_{\varphi}^{T}(q)\right)^{-1} J_{\varphi} \hat{S}_{q}\right]$ If $\gamma_{F}>\varepsilon_{5}$, then a sliding mode at $S_{F \delta}(t)=0$ is induced in a time $t_{f} \leq \frac{\left|S_{F \delta}\left(t_{0}\right)\right|}{\mu_{F}}$, but $S_{F \delta}\left(t_{0}\right)=0$.

Part III: Exponential convergence of tracking errors.

Part III.a: Visual tracking errors. Since a sliding mode exists for all time at $S_{s \delta}(t)=0$, then, we have that $S_{s}=S_{s d} \forall t \rightarrow \Delta \dot{x}_{s}=$ $-\alpha \Delta x_{s}+S_{s}\left(t_{0}\right) e^{-\kappa_{s} t}$. This implies that the visual tracking errors locally tends to zero exponentially fast, this is $x_{s} \rightarrow x_{s d}, \dot{x}_{s} \rightarrow \dot{x}_{s d}$, implying that the robot end-effector converges to the desired image $x_{s d}$, with given velocity $\dot{x}_{s d}$.

Part III.b: Force tracking errors. Since a sliding mode at $S_{F \delta}(t)=0$ is induced for all time, this means $\Delta F=\Delta F\left(t_{0}\right) e^{-\kappa_{F} t}$. Moreover, in (V. Parra-Vega, 2003) it is showed that the convergence of force tracking errors arises, thus $\lambda \rightarrow \lambda_{d}$ exponentially fast. QED.

Remark 2. We have proved that $J(q(t))$ is not singular for all time, because $q(t) \rightarrow q_{d}(t)$ exponentially, without overshoot, with desired trajectories belonging to robot workspace $\Omega$, thus $J(q(t)) \rightarrow J\left(q_{d}(t)\right)$ within $\Omega$.

Remark 3. Using continuous tanh $(*)$ instead of $\operatorname{sign}(*)$ induces larger upper bounds $\varepsilon_{2}$ and $\varepsilon_{3}$ in comparison of using sign $(*)$, with the great advantage of getting ride of the harmful chattering. In this case, to induce the second order sliding mode, and therefore exponential convergence of tracking errors, it suffices to tune $\gamma_{F}$ and $\gamma_{P}$ to a larger value.

\section{DYNAMIC FRICTION COMPENSATION}

Now let us consider the dynamic friction into the model, which represent a very realistic behaviour when the robot is moving along a rigid surface, in particular, driven by visual servoing. In this case, the following LuGre (Canudas de Wit and Astrom, 1995) dynamic friction model is very appropiate to define the joint friction

$$
\begin{aligned}
F(\dot{q}, \psi) & =\sigma_{0} z+\sigma_{1} \dot{z}+\sigma_{2} \dot{q} \\
\dot{z} & =-\sigma_{0} h(\dot{q}) z+\dot{q} \\
h(\dot{q}) & =\frac{|\dot{q}|}{\alpha_{0}+\alpha_{1} \exp ^{-\left(\dot{q} / \dot{q}_{s}\right)^{2}}}
\end{aligned}
$$

where matrix parameters $\sigma_{1}, \sigma_{2}, \sigma_{3}$ are diagonal definite matrices $n \times n$, the state $z \in R^{n}$ stands for the position of the bristles, $\alpha_{0}, \alpha_{1}>0$, and $\dot{q}_{s}>$ 0 . This model involve a very complex dynamics around the trivial equilibrium, for bidirectional motion, and for very small displacements, the forces that comes out from this model makes impossible to reach the origin due to limit cycles induced and the potentially unstable behavior. Substituting (30) into (1) yields

$$
\begin{aligned}
& H(q) \ddot{q}+C(q, \dot{q}) \dot{q}+J(q)^{T} B_{t} J(q) \dot{q}+\sigma_{12} \dot{q}+ \\
& g(q)+\sigma_{0} z-\sigma_{01} h(\dot{q}) z=\tau+J_{\varphi}^{T}(q) \lambda
\end{aligned}
$$

where $\sigma_{01}=\sigma_{0} \sigma_{1}$ and $\sigma_{12}=\sigma_{1}+\sigma_{2}$. Now, we need to organize the parametrization in terms of two regressors: $H(q) \hat{\ddot{q}}_{\text {cont }}+\left(C(q, \dot{q})+J(q)^{T} B_{t} J(q)+\right.$ $\left.\sigma_{12}\right) \hat{\dot{q}}_{r}+g(q)=Y_{\text {cont }} \Theta_{b}$ and the virtual continuous regressor $\frac{\sigma_{01} \alpha_{01}}{\alpha_{0}}|\dot{q}| \tanh \left(\lambda_{f} S_{q}\right)+\sigma_{0} \alpha_{01} \tanh \left(\lambda_{f} S_{q}\right)=$ $Y_{f} \Theta_{f}$, where $\alpha_{01}=\alpha_{0}+\alpha_{1}, \tanh (q)$ is the continuous hyperbolic tangent function, and $\lambda_{f}>0$. Now, If we add and subtract the above regressors to (31) yields the following open-loop error dynamics with dynamic friction

$$
\begin{aligned}
H(q) \hat{\dot{S}}_{q} & =-\left(C(q, \dot{q})+J(q)^{T} B_{t} J(q) \hat{S}_{q}+\sigma_{12}\right) \hat{S}_{q}+ \\
& -\mathcal{F}-Y \Theta+J_{\varphi}^{T}(q) \lambda-\sigma_{0} z+\sigma_{01} h(\dot{q}) z \\
& +H\left(Q \gamma_{s} z_{s}-\beta J_{\varphi}^{T} \gamma_{f} z_{f}\right)
\end{aligned}
$$

where $\mathcal{F}=\sigma_{0} z+\sigma_{0}+\sigma_{0} \alpha_{0}{ }^{-1} \sigma_{1}|\dot{q}|-\sigma_{0} \sigma_{1}|\dot{q}| z \alpha_{e}$, $\sigma_{x}=\alpha_{01} \tanh \left(\lambda_{f} S_{q}\right), \alpha_{e}=\left(\alpha_{0}+\alpha_{1} \exp ^{-\left(\dot{q} / \dot{q}_{b}\right)^{2}}\right)^{-1}$, $Y=\left[Y_{\text {cont }}, Y_{f}\right]$, and $\Theta=\left[\Theta_{b}^{T}, \Theta_{f}^{T}\right]^{T}$. Finally, consider the following control law

$$
\begin{aligned}
\tau & =-K_{d} \hat{S}_{q}+Y \hat{\Theta}_{b}+J_{\varphi}^{T}(q)\left[-\lambda_{d}+\eta \Delta F\right] \\
& +\gamma_{F} J_{\varphi}^{T}(q)\left[\tanh \left(\mu S_{q F}\right)+\eta \int \operatorname{sgn}\left(s_{F \delta}\right)\right] \\
\dot{\hat{\Theta}} & =-\Gamma Y^{T} \hat{S}_{q}
\end{aligned}
$$

where $\Gamma \in R_{+}^{p \times p}, K_{d} \in R_{+}^{n \times n}, \gamma_{F}>0, \eta>0$. We now have the following result.

Theorem 2. Consider the constrained robot (1) subject to parametric uncertainty on the robot, the camera, tangential contact friction and joint dynamic friction. Assume that initial conditions and desired trajectories belong to $\Omega$, y consider the adaptive visual servoing force-position controller (33)-(34). If $K_{d}$ is large enough and a error of initial conditions are small enough, and if $\gamma_{s} \geq$ $\left\|\frac{d}{d t}\left\{R_{\alpha}(\theta) J(q)\left[\hat{S}_{q}+\left(\widehat{J_{\text {Rinv }}}-J_{\text {Rinv }}\right) \dot{x}_{r}\right]\right\}\right\|$ and 
$\gamma_{F} \geq\left\|\frac{d}{d t}\left[\left(J_{\varphi} J_{\varphi}^{T}(q)\right)^{-1} J_{\varphi} \hat{S}_{q}\right]\right\|$ then exponential convergence of visual and force tracking errors is guaranteed.

Proof.- With the very same Lyapunov function of theorem 1, we obtain the time derivative, along trajectories of the closed loop of (33)-(34) and (1), as: $\dot{V} \leq-\lambda_{\min }\left(K_{d}\right)\left\|\hat{S}_{q}\right\|_{2}^{2}-\eta \beta\left\|S_{v F}\right\|+\left\|\hat{S}_{q}\right\| \psi-$ $\dot{V}_{f}$, where $\dot{V}_{f}=\sigma_{0} S_{r}^{T}\left[z+\sigma_{01} \tanh \left(\lambda_{f} S_{r}\right)\right]-$ $\sigma_{01} S_{r}\left[-z h(\dot{x})+\alpha_{0}{ }^{-1} \sigma_{01}|\dot{x}| \tanh \left(\lambda_{f} S_{r}\right)\right] .>0$, see (Garcia-Valdovinos and Parra-Vega, 2003). From here on, we proceed exactly as in proof of Theorem 1, details are therefore omitted. QED.

\section{SIMULATIONS}

Robot parameters are taken from a 2 DOF planar robot, also the camera parameters are from SONY DFWVL500 CCD camera. Robot parameters: Mass $(6,2) \mathrm{kg}$, Length(0.4,0.3)m. Camera: $\theta\left(90^{\circ}\right), \alpha(77772) p i x / m, z(1.5) m$. Friction parameters: $\sigma_{0}(30000), \sigma_{1}=\sigma_{2}(2), \alpha_{0}(4), \alpha_{1}(0.4)$, $\dot{q}_{s}(0.01)$. The desired trajectories for the simulation is $x_{s}=\alpha R[x c d ; y c d]+\beta, x c d=0.5 ; y c d=$ $0.5+r * \sin (\omega * t) ; r=0.1, \omega=0.5$. The contact surface is a plane parallel to plane $Y Z$ and over $x=0.5$.

Feedback gains are $\Gamma=\operatorname{diag}(1), \kappa_{f}=20, \gamma_{f}=$ $3.0, \eta=0.029, \beta=1.0, K_{d}=\operatorname{diag}(90), \alpha=$ $\operatorname{diag}(40), \kappa s=20, \gamma_{s}=\operatorname{diag}(7.8)$.

\section{CONCLUSIONS}

A novel adaptive servo visual scheme for a constrained dynamical robot system is presented. The main feature of this scheme is the ability to fuse image coordinates and contact forces. Local exponential convergence arises for image positionvelocity and contact forces even when robot parameters, camera parameters and contact friction are unknown. Additionally, it is proposed a compensator of uncertain joint dynamical friction, which has never treated in the literature of visual servoing, but it is particularly important in contact motion tasks, because the motion regime is slow, with velocity reversals. Simulations confirm the predicted stability properties.

\section{REFERENCES}

Arimoto, S. (1996). Control theory of non-linear mechanical systems. Clarendon Press, Oxford.

Canudas de Wit, C., Olsson H. and K.J. Astrom (1995). A new model for control of systems with friction. IEEE Transactions of Automatic Control 40(3), 419-425.
Garcia-Valdovinos, L. G. and V. Parra-Vega (2003). Experimental issues of finite time compensation of dynamic friction for robots. American Control Conference, Denver, USA.

J. Baeten, W. Verdonck, H. Bruyninckx J. De Schutter (2000). Combining force control and visual servoing for planar contour following. Machine Intelligence and Robotic Control 2(2), 69-75.

S. Hutchinson, G. Hager, P. Corke (1996). A tutorial on visual servo control. Trans. on Robotics and Automation.

V. Parra-Vega, S. Arimoto, Y. Liu G. Hirzinger P. Akella (2003). Dynamic sliding PID control for tracking of robot manipulators: Theory and experiments. IEEE Transactions in Robotics and Automation.

Xiao, D. (2000). Sensor-hybrid Position/Force control of a robot manipulator in an un calibrated enviroment. IEEE Trans. Control Systm Technology 8(4), 635-645.
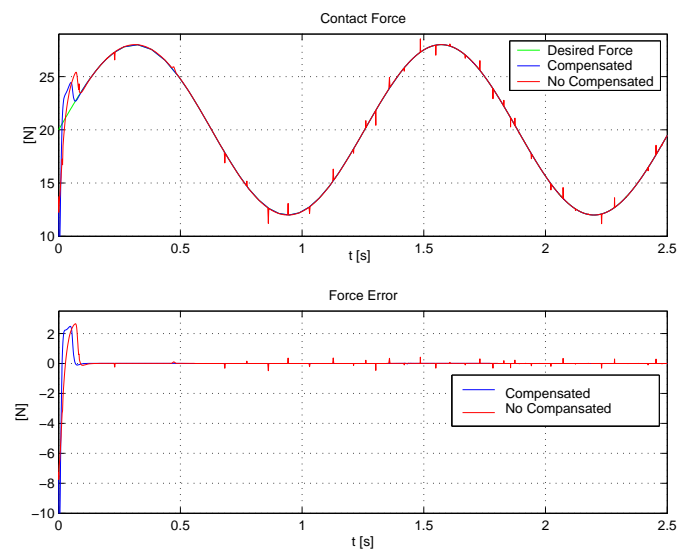

Figure 2. Force tracking errors indicate very fast convergence. As we can see, without friction compensation the force tracking exhibits overshot.
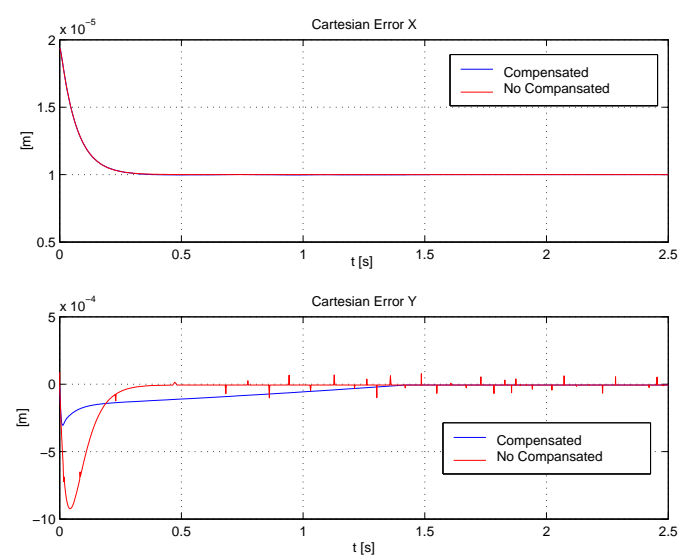

Figure 3. Cartesian tracking error with exponential envelope. Once again, the visual tracking presents overshots when no friction compensation is available. 